\title{
Diálogos entre el feminismo postestructuralista y la teoría de la interseccionalidad de los géneros
}

\author{
Dialogue about feminism post-structuralist and theory of \\ intersectionality of gender
}

Dra. Laura Zambrini ${ }^{7}$

\begin{abstract}
"Lo que resulta problemático en este uso de "mujeres" como grupo, como categoría de análisis estable, es que se asume una unidad antihistórica y universal entre las mujeres, fundada en la noción generalizada de su subordinación. En

vez de demostrar analíticamente la producción de las mujeres como grupos socioeconómicos y políticos dentro de contextos locales particulares, esta jugada analítica limita la definición del sujeto femenino a la identidad de género, ignorando por completo identidades de clase o étnicas".
\end{abstract}

(Tapalde Mohanty, 1988: 12)

\begin{abstract}
Resumen
El objetivo principal del trabajo es reflexionar sobre la puesta en diálogo entre dos vertientes teóricas que influenciaron el campo de los estudios de género: el postestructuralismo y la perspectiva de la interseccionalidad. Cada una, desde su particular mirada, propone una visión de las nociones de sujeto e identidad femenina. Se plantea que dichas visiones no son discordantes, por el contrario, ambas se complementan y logran un análisis certero de las problemáticas de género actuales, en especial en contextos latinoamericanos.
\end{abstract}

Palabras clave: postestructuralismo - interseccionalidad - géneros - feminismo - poscolonial.

\begin{abstract}
The main objective of this paper is to dialogue about two aspects that influenced the gender studies: post-structuralism and intersectionality perspectiva. Each one, from their particular look, proposes a view of subject and female identity. Here, we propose that these visions are not discordant; on the contrary, they complement and achieve an accurate analysis of the current gender issues in latinoamerican contexts.
\end{abstract}

Key words: post-structuralism - intersectionality - gender - feminist - postcolonial.

Fecha de recepción: 27 de mayo de 2013

Fecha de aprobación: 9 de septiembre de 2014

\footnotetext{
Laura Zambrini es Socióloga (UBA) y Doctora en Ciencias Sociales (UBA). Dicta clases como profesora titular en la cátedra de Sociología en la Universidad de Buenos Aires, Argentina (FADU-UBA), donde también dirige un proyecto de investigación SI-FADU. Es becaria posdoctoral del CONICET.

Universidad de Buenos Aires-CONICET.
} 


\section{INTRODUCCIÓN}

El objetivo principal del presente trabajo es reflexionar acerca de la puesta en diálogo de dos vertientes teóricas que influenciaron el campo de los estudios de género: el feminismo postestructuralista y la perspectiva de la interseccionalidad. Cada una, desde su mirada, propone una visión particular sobre las nociones de sujeto e identidades femeninas. Aquí, planteamos que dichas visiones no son discordantes, por el contrario, creemos en la pertinencia de su articulación y complementariedad para un análisis certero de las problemáticas de género en los escenarios poscoloniales, por ejemplo en Latinoamérica hoy día. En este sentido, a lo largo del artículo, en primer lugar, se realiza un breve repaso por los fundamentos del postestructuralismo y su peculiar encuentro con el pensamiento feminista (en particular, acerca de los usos sociales del lenguaje y su potencialidad para crear realidades de manera performativa); en segundo lugar, se exponen los fundamentos de la teoría de la interseccionalidad de los géneros y su singular aporte sobre el cruce de categorías como género, racismo y poder. Y por último, se destacan los puntos de contacto entre ambas teorías en virtud del robustecimiento de la lucha feminista en los actuales contextos poscoloniales. Esto es, los aportes del feminismo postestructuralista posibilitaron caracterizar las identidades de género como construcciones preformativas y la interseccionalidad ha posibilitado pensar la consolidación de la modernidad como un proceso inherente al colonialismo discursivo, inclusive en el campo académico. Es decir, asumir la negación y el oscurecimiento histórico de otras formas de pensamientos, vidas y conocimientos en virtud de la construcción de otredad/es y alteridades.

Actualmente, algunas vertientes del campo académico (en especial, los estudios de género) han incorporado la perspectiva de la interseccionalidad entre las categorías de clase, raza, etnia, género para dar cuenta de la situación de opresión de las minorías silenciadas por el mundo occidental. La perspectiva de la interseccionalidad como marco teórico nos habilita a rendir cuenta de la configuración cruzada de las relaciones de poder. Por un lado, denuncia el proceso histórico de colonización discursiva que ha operado arbitrariamente, también en el campo académico mediante el uso de categorías con las cuales se intenta producir conocimiento, pero producidas en otros contextos. Y por otro lado, evidencia cómo los discursos con fines emancipatorios (tal como el discurso feminista), también pueden contener implícitamente exclusiones no dichas en su interior.

\section{PENSAMIENTO BINARIO E IDENTIDADES FEMENINAS}

Para comenzar con el recorrido propuesto debemos mencionar que para el campo de los estudios de género, el pensamiento de M. Foucault ha sido una pieza fundamental. Entre otras cuestiones, la conceptualización de la sexualidad como un dispositivo demostró la importancia que ha tenido el siglo XIX, momento en que la sexualidad humana adquirió estatus de objeto de estudio científico (Foucault, 2003). Básicamente, el auge del modelo de ciencia positivista (como forma privilegiada de conocimiento y explicación del mundo) se apoyó en un sistema clasificatorio que caracterizaba los 
fenómenos estudiados como normales o patológicos. En lo que atañe al orden de los sexos y las prácticas, esa clasificación auspició la consolidación de la heterosexualidad como el punto de referencia desde cual se calificaba el resto de las conductas, logrando imponer la heterosexualidad reproductiva como la normativa obligatoria. Estas premisas pueden ser complementadas desde la disciplina antropológica por G. Rubin (1993), quien entendió que, en esa misma etapa, se consolidó un sistema sexo/genérico: el enlace a nivel ideológico del sexo con la naturaleza y el género con la cultura. Si bien, Foucault no tuvo en cuenta de manera explícita la perspectiva de género, a nivel conceptual, tanto el dispositivo de la sexualidad como el sistema sexo/ género, coinciden en destacar que en el siglo XIX se naturalizó el pensamiento binario como el principal ordenador de la vida social moderna.

En el ámbito de los géneros y las sexualidades, los principales binarismos (excluyentes entre sí por definición) que se impusieron fueron: hombre/mujer, masculino/femenino y heterosexual/homosexual. Estos establecieron la normativa hegemónica a partir de la naturalización de una supuesta coherencia entre el sexo, el género, la presentación corporal, las emociones, los roles sociales, entre otras. En esa directriz, las prácticas no heterosexuales fueron fuertemente estigmatizadas a partir del proceso histórico que consolidó en la modernidad al pensamiento binario. Desde esta perspectiva moderna, las identidades eran explicadas gracias al orden de la naturaleza, dejando de lado las mediaciones sociales, culturales e históricas. El género fue entendido como una categoría natural, cerrada e inmóvil. Sin embargo, el binomio femenino/masculino, además de ser excluyente, ha contenido jerarquías en su interior. Lo masculino se impuso históricamente como dominante y relegó lo femenino como categoría subalterna. Lo masculino fue asociado a lo productivo, al poder, la racionalidad, la fuerza y lo público. En cambio, lo femenino se enlazó a la reproducción, lo débil, las emociones y lo privado. En este sentido, las feministas fueron quienes, a partir de sus singulares articulaciones entre la teoría y la política, han denunciado la opresión que atañe a las mujeres y al universo femenino en el patriarcado. Paradójicamente, y como se verá luego, esas voces feministas se alzaron en virtud de la emancipación de las mujeres blancas y burguesas, dejando de lado otras experiencias como las vivencias de las mujeres negras, indígenas o migrantes, por ejemplo. En ello radica una de las críticas más sustantivas hacia gran parte de la producción teórica del feminismo occidental.

\section{LA TERCERA OLA FEMINISTA Y LA INFLUENCIA DE LA DECONSTRUCCIÓN DE JACQUES DERRIDA}

Como se señaló, el feminismo occidental articuló lo académico y lo político para discutir el lugar subalterno que las mujeres tenían en la configuración del orden social moderno caracterizado por la supremacía de lo masculino y el patriarcado. El feminismo no puede ser caracterizado como un movimiento social homogéneo ni como un cuerpo teórico uniforme. En su historia, los distintos marcos conceptuales y políticos con los que se ha pensado la relación de poder entre los sexos/géneros dieron lugar a diferentes interpretaciones y posturas. 
A modo de síntesis, suele conceptualizarse el recorrido del pensamiento feminista en términos de "olas" (Gamba, 2007). La"primera ola"feminista está definida por el movimiento sufragista y ciudadano surgido con posterioridad a la Revolución Francesa. Olympia de Gouges en Francia y Mary Wollstonecraft en Inglaterra se consideran sus principales referentes (Gamba, 2007). Los aportes fundamentales residen en la lucha dada para que las mujeres fueran incluidas en la ciudadanía, ya que esta solo era definida en términos masculinos.

La "segunda ola", cuya mayor representante fue Simone de Beauvoir, intentó desnaturalizar la categoría mujer para pensarla como una categoría social (De Beauvoir, 1949). A partir del lema lo personal es político se logró cuestionar el patriarcado, la división sexual del trabajo, el trabajo doméstico y la separación de los mundos públicos y privados. La redefinición de la identidad personal de las mujeres era considerada fundamental para obtener un cambio político (Gamba, 2007).

La "tercera ola"feminista partió de preguntarse acerca de quién/quiénes eran los sujetos políticos del feminismo y a quiénes representaba. Esta vertiente considera al cuerpo femenino como una producción cultural e histórica, trascendiendo la típica dualidad varón/mujer (Braidotti, 1999). Desde esta óptica, lo femenino y lo masculino son caracterizados como construcciones sociales. Se desmonta la relación sexo/género a partir de la incorporación del giro performativo propuesto por J. Butler para pensar las relaciones históricas de género (Austin, 1992 y Butler, 1997). Precisamente, a partir de la impronta de la tercera ola, es cuando se afianza el encuentro entre el feminismo y el pensamiento filosófico postestructuralista (Zambrini \& ladevito, 2009). Con ello se trastocan los modos de pensar las nociones de sujetos e identidades en sentido amplio y se renuevan los planteos del feminismo. En esa etapa de los estudios de género, las teorías de J. Derrida y M. Foucault fueron centrales, porque facilitaron la historicidad del pensamiento binario al cuestionar las estructuras propias de la racionalidad moderna. Una racionalidad basada en una concepción universalista del ser humano y que, a su vez, es fundante de una noción de sujeto regida por una lógica binaria de la identidad, como se expuso anteriormente. Dicha lógica se denomina el paradigma de las identidades y fue la que sostuvo la supuesta correspondencia entre el mundo y el lenguaje.

Asimismo, desde el punto de vista de los estudios de género, el paradigma de las identidades estableció como natural la relación entre el sexo y la corporalidad, fortificando la matriz heterosexual como la norma social dominante.

Efectivamente, el postestructuralismo auspició la ruptura con ese paradigma de las identidades, abriendo paso a la pregunta por las identificaciones. Este pasaje se produjo gracias a la incorporación del denominado giro performativo en las ciencias sociales (Austin, 1962) puesto que posibilitó la deconstrucción de categorías como hombre, mujer, femenino, masculino, etc.; un salto cualitativo que dio lugar desde la teoría a la puesta en duda de aquellas visiones herméticas de las concepciones de sujeto e identidad. Es decir, ya no resultaba pertinente pensar al sujeto como una esencia, 
sino que a partir de esta renovación teórica se piensa la subjetividad atravesada por múltiples dimensiones/posiciones que la configuran, como la edad, la clase social, etnia, sexo, género, etc. Justamente, dicho cambio conceptual en el modo de concebir al sujeto, es lo que sustenta la propuesta de este artículo sobre la puesta en diálogo del feminismo postestructuralista y la teoría de la interseccionalidad de los géneros.

Esto es, el género como tal se entrecruza con múltiples dimensiones sociales y políticas que crean y recrean la vida social. Esa caracterización se afinca en una crítica profunda a la racionalidad moderna e ilustrada, considerada la columna vertebral de la cultura occidental y del pensamiento binario, dando lugar a aquellas voces silenciadas por los saberes occidentales como las surgidas en las teorías poscoloniales.

\section{JUDITH BUTLER: GÉNERO Y DISCURSO}

Para profundizar lo expuesto en el punto anterior, corresponde destacar que la tercera ola feminista posibilitó la incorporación de autores que no necesariamente pensaron sus teorías y conceptos en función de las problemáticas de género. Sin embargo, sus aportes fueron muy enriquecedores para este campo, por ejemplo, autores como J. Derrida (1971), J. Lacan (1977) y S. Hall (1996) han trabajado en torno al concepto de "inestabilidad". Este concepto define las construcciones identitarias como los usos mismos del lenguaje. Ello significa que mediante el lenguaje no es posible fijar/clausurar el significado de un modo final y para siempre. De igual manera, no es viable pensar la identidad como fijada/anclada, sino que la misma se construye permanentemente en un proceso dinámico y abierto. Estas premisas fueron retomadas por J. Butler (2001) para trabajar en particular la cuestión de los géneros.

Entre otros aspectos, la autora tomó en cuenta el término "deconstrucción"introducido en la década del 60 por J. Derrida (1989). La deconstrucción expresaba cierto interés por las estructuras, al tiempo que sugería cuestionarlas. No obstante, la deconstrucción se trata de un acontecimiento que no espera la conciencia u organización del sujeto. En este sentido, mediante la noción de différance, que propone mirar la temporalidad y establecer el acento en la falta de identidad, Derrida sostuvo que el sentido es siempre algo diferido, característica que también afecta al lenguaje y al sujeto respecto de sí mismo (Arfuch, 2002). Ello permite concebir al sujeto como una multiplicidad abierta y a las identidades como ficciones que se reinventan nuevamente en cada contexto. Esa diversidad expresada como singularidad de lo deconstruido nos estimula a plantear las deconstrucciones en plural (Derrida, 1971). Según esta perspectiva teórica, el lenguaje está signado por la inestabilidad, pero al mismo tiempo se sugiere que las identidades también son inestables porque se construyen culturalmente por ese mismo lenguaje indeterminado y constitutivo de toda relación social.

Bajo el influjo postestructuralista, el concepto de performatividad trabajado por J. Butler ha sido fundamental en su caracterización de las identidades de género. No obstante, para una comprensión adecuada del pensamiento de J. Butler, es preciso tener en cuenta la contribución teórica de J. Austin sobre el habla y el discurso. Este autor 
distingue las formaciones lingüísticas que describen un estado de cosas (emisiones constatativas) de aquellas que son acciones (emisiones performativas) y señala que estas últimas -por el hecho mismo de expresar- están dando cuenta de una acción, lo que no significa que describan lo que el sujeto está haciendo (Austin, 1992).

La idea de lo performativo que piensa J. Austin sobre el lenguaje es coincidente con el modo en que J. Butler concibe los géneros (como performances). Como se indicó, Butler toma en cuenta para el desarrollo de sus ideas la concepción deconstructiva del lenguaje de J. Derrida y sus conceptos de différance y huella, que ponen el énfasis en la ausencia, la distancia y la diferencia entre el objeto y su representación.

J. Butler concuerda con J. Derrida que en la expresión de una emisión, experiencia, actuación se está realizando una acción performativa, enfatizando la dimensión discursiva del sujeto y lo social (Butler, 1997 y 2001).

J. Butler desarrolló la perspectiva de la performatividad de los géneros y propuso cuestionar lo binario y la naturalización de la normativa hegemónica heterosexual. Ella define lo performativo como la reiteración de las prácticas discursivas en torno a la relación sexo/género en tanto categorías contingentes. Mediante su crítica fundamenta que es precisamente a partir de la reiteración discursiva que se produce la materialización de los cuerpos y de las identidades de acuerdo con la norma heterosexual. Sostiene, asimismo, que por medio del lenguaje se define en el ámbito político a quiénes se representa y a quiénes se excluye (Butler, 1997 y 2001).

La autora retoma una noción semiótica destacada por J. Derrida respecto del carácter repetitivo e iterable que deben tener los signos para ser considerados como tales. Lo mismo ocurre con el lenguaje y su posibilidad de ser citado para la creación de sentido, es decir, la performatividad del habla. A partir de la combinación entre las operaciones citacionales, iterables y performativas del lenguaje es que llegan a producirse las identidades culturales, y por consiguiente, las identidades de género. Por ende, los sujetos se vuelven inteligibles socialmente en la reproducción de las normas. En otras palabras, el sexo y el género son interpretados como construcciones culturales.

En esta directriz resulta imposible plantear al cuerpo como un espacio neutro o previo al sentido. Es decir, las posibilidades corpóreas se encuentran condicionadas y mediadas por el discurso social normativo. No obstante, J. Butler afirma que mediante la actuación y las performances pueden ser modificadas, subvertidas o al menos, cuestionadas. La supuesta estabilidad que surge de la coherencia ficcional entre el sexo/género es atravesada por múltiples relaciones sociales tendientes a normalizar la heterosexualidad como obligatoria. J. Butler sostiene que de la dinámica entre la elección/coacción surge la posibilidad de invención aunque no son elecciones/invenciones radicales, sino las potenciales dentro de un horizonte cultural determinado. J. Butler, retomando el ya clásico planteo de S. de Beauvoir "no se nace mujer, sino que se hace", afirma que "no se nace" con un determinado género, sino que el mismo "se hace" en un proceso sin fin que se sostiene en la reiteración de las prácticas, en una 
constante lucha y negociación con el poder y las normas socioculturales (De Beauvoir, 1949; Butler, 2001).

En síntesis, la performatividad es retomada por Butler (a partir de las teorías del discurso) para repensar los géneros como formas mediante las cuales las palabras conllevan acciones. Estas acciones muchas veces se manifiestan en actos machistas y sexistas que convierten a las mujeres de los diversos contextos, o todo aquel que no acate las normas, en víctimas de violencia física y/o simbólica. De ahí la necesidad de repensar la discriminación de género en sintonía con otros dispositivos afines al racismo como la clase social y la raza/etnia. Asimismo, en el campo académico surgen nuevas preguntas respecto de los usos de los conceptos como instrumentos políticos, así como también de los vínculos entre las teorías y los contextos en los que se aplican (esto es, la colonialidad de las relaciones de género y los conceptos con los que se las piensa).

Con todo, una crítica feminista acertada nos habilitaría a poder enunciar las múltiples diferencias de las mujeres que se entrecruzan de modo constante e interseccional. Es decir, entender el género como un conjunto de relaciones móviles, dinámicas, variables y en transformación permanente.

\section{GÉNERO E INTERSECCIONALIDAD}

A partir de lo expuesto en el apartado anterior y siguiendo a J. Butler, podemos establecer que la vida social está "generizada" porque el género como tal entrecruza y atraviesa otras dimensiones sociales y políticas. Ahora bien, este trabajo se propuso como objetivo general articular esas premisas con la perspectiva de la interseccionalidad de los géneros ¿Por qué? En primer término, porque esta perspectiva propone interpretar el racismo y el sexismo como fenómenos que operan mediante dispositivos que resultan afines (Davis, 1981). Para una mayor claridad expositiva podemos decir que esta argumentación se sustenta en las siguientes proposiciones:

a) El racismo y sexismo apelan al orden de la naturaleza para justificar relaciones de poder.

b) Ambos asocian relaciones que comprometen a los cuerpos con relaciones sociales más amplias.

c) Tanto el racismo como el sexismo son representaciones inscritas en relaciones sociales, políticas y culturales. No es posible pensar las clases sociales, la sexualidad, la raza, entre otros, sin atender a la dimensión de género.

d) El racismo y el sexismo operan políticamente en sistemas de opresión interconectados que resultan muy difíciles de distinguir.

Atendiendo estas cuestiones, K. Crenshaw recreó el concepto de interseccionalidad cuyo fin es comprender la convergencia de las distintas formas de discriminación padecidas por las mujeres ligadas al sexismo, clasismo y racismo (Crenshaw, 1994). En el ámbito teórico, la perspectiva de la interseccionalidad de los géneros nos invita a entrecruzar distintas categorías de análisis que posibiliten la comprensión de los complejos 
mecanismos que intervienen en la cuestión de los géneros. La interseccionalidad propone un análisis relacional entre las clases sociales, las etnias/razas, las sexualidades, los géneros, entre otros aspectos (Tapalde Mohanty, 1988; Crenshaw, 1994). Si bien, esta categoría surgió de las mujeres afroamericanas para pensar su propio contexto, dando lugar al surgimiento de la corriente conocida como black feminism (Davis, 1981), a su vez, esta perspectiva ha posibilitado establecer las relaciones adecuadas para entender las formas de desigualdades sociales que operan con dispositivos afines, como el sexismo, el clasismo o el racismo, que intervienen de manera combinada en la distribución del poder en los contextos locales (Viveros, 2002 y 2006).

La perspectiva de la interseccionalidad de los géneros habilita a dar cuenta de la configuración cruzada de las relaciones de poder. Según M. Viveros, en América Latina el género ha sido asociado a las clases sociales; en contraste, el feminismo negro en los Estados Unidos se concentró en la articulación entre el género y la raza (Viveros, 2002). Sin embargo, este enfoque resulta oportuno para pensar los actuales debates en las ciencias sociales en torno a las nociones de diferencia, diversidad, pluralidad, multiculturalismo, poscolonialismo. Aquí, consideramos pertinente la puesta en diálogo con el feminismo postestructuralista, porque la interseccionalidad, en primer lugar incluye en su análisis a los distintos sistemas de opresión sociales. En segundo lugar, incorpora la denuncia realizada por J. Butler respecto del sesgo heteronormativo del concepto de género. Y en tercer lugar, se apoya en la existencia de múltiples experiencias del sexismo articuladas en múltiples formas de discriminación social (Butler, 1997; 2001; 2004 y 2006).

Para C. Tapalde Mohanty (1988), desde las posiciones marginales de sujeto es posible cuestionar la construcción de las posiciones dominantes. Pero, a su vez, hablar de interseccionalidad supone hacer una elección teórica respecto de cuáles categorías se consideran relevantes para explicar la confluencia de determinadas relaciones de poder. Entonces, resulta determinante declarar desde qué lugar epistémico se hace esta elección. Es decir, el uso indiscriminado de la puesta en relación de categorías como la raza, género y clase, entre otras, no necesariamente serán apropiadas para analizar todos los contextos, como por ejemplo el latinoamericano.

Como ya se dijera anteriormente, el pensamiento feminista no puede pensarse como un cuerpo teórico unificado, no obstante, las distintas vertientes teóricas feministas tienen como denominador común el cuestionamiento de una de las matrices más importantes de la cultura moderna y occidental: el patriarcado. A pesar de que existen distintas vertientes feministas, a los efectos de este trabajo nos interesa destacar que el acento puesto exclusivamente en el patriarcado opacó por mucho tiempo las diferencias subjetivas de las propias mujeres de los distintos contextos. Es decir, la opresión del patriarcado no es ni ha sido una experiencia homogénea ni universal. En este sentido, C. Tapalde Mohanty (1988) ha demostrado que la voz dominante del feminismo concentrada en el patriarcado como la causa principal de la opresión femenina construyó, por un lado, la categoría "mujer del tercer mundo" y por otro, la noción de "occidente". Si bien, ambas categorías teóricas son ficciones imaginarias, 
también tienen efectos concretos en la praxis social. En este punto, la autora establece una relación compleja entre la colonización y los discursos. Esto es, una dominación más amplia que anula la heterogeneidad ya desde el lenguaje mismo. Por ejemplo, la categoría "mujer del tercer mundo", además de homogeneizar a los sujetos que intenta representar podemos decir que también tiene como finalidad sutil la creación de la categoría "mujer del primer mundo". De este modo, occidente se construye como el punto de referencia principal desde donde se define a la otredad, como lo afirmó F. Fanon (1963) en su clásica crítica al eurocentrismo.

Por varias de estas razones, C. Tapalde Mohanty (1988) denuncia el proceso histórico de colonización discursiva que ha operado arbitrariamente también en el campo académico mediante el uso de categorías con las cuales se intenta producir conocimiento y teoría. En palabras de K. Bidaseca: Es el feminismo liberal burgués y occidental hegemónico, cuyo emblema es la mujer blanca, occidental, heterosexual, de clase media, urbana, educada y ciudadana contra el patriarcado como categoría universal, el que es cuestionado por otras mujeres que observan ciertas ausencias sintomáticas en la agenda feminista: el racismo, lesbofobia, la colonización. Al llamar a la unidad del feminismo para luchar contra la opresión universal del patriarcado, las feministas -que desconocían la opresión de raza y clase-pospusieron y desecharon estas otras opresiones y, de este modo, impidieron ver sujetos racializados, sexualizados y colonizados y su ubicación en diferentes discursos racializados (Bidaseca, 2010: 131).

La cita precedente nos da cuenta de la necesidad de la deconstrucción de ciertas categorías como la de género, por ejemplo. Como ya hemos dicho, la perspectiva de la interseccionalidad surgió desde el feminismo negro para enfrentar el silenciamiento de las mujeres negras producido por la homologación de la categoría mujer con el feminismo dominante blanco y burgués (Davis, 1981). La interseccionalidad ha sido afín por un lado, a las teorías producidas en los Estados Unidos en torno a las políticas de la identidad; y por otro, al postestructuralismo francés. No obstante, aquí se desea alertar que la incorporación de esta perspectiva puede conllevar a usos inadecuados o contradictorios de dicho concepto. Por ejemplo, al plantear las relaciones sociales que se pretenden analizar como estructuras estables, recayendo en una suerte de esencia de la dominación. Como se ha expuesto a lo largo del artículo y acorde a la marca del postestructuralismo, las relaciones sociales están en constante cambio y movimiento. Sin embargo, la perspectiva de la interseccionalidad puede sernos útil para separar analíticamente dimensiones que en lo fáctico jamás podrían pensarse por separado. Las relaciones sociales generan experiencias dinámicas que no pueden distinguirse tan fácilmente como la raza, el género o la clase social. Las mismas operan simultáneamente y solo es posible diferenciarlas para su análisis.

Quedará abierto el interrogante respecto de la posibilidad de construir un conocimiento situado desde una mirada feminista latinoamericana con voz propia, que parta desde las experiencias de vida de las mujeres subalternas latinoamericanas: indígenas y campesinas, afrodescendientes, migrantes, lesbianas, trabajadoras en las maquilas, trabajadoras sexuales, transgéneros, entre otras. Asumimos que esas experiencias 
subalternas poseen componentes identitarios que responden a realidades y contextos diferentes. Sin embargo, a pesar que el postestructuralismo y la interseccionalidad han brindado las herramientas conceptuales apropiadas, no puede dejar de señalarse la ausencia que han tenido dichas voces durante mucho tiempo en la producción teórica del feminismo occidental.

\section{PALABRAS FINALES}

En los últimas décadas, la corriente postestructuralista ha colaborado en el trazado de políticas de la identidad y la diferencia (Fraser, 1997) en tanto estrategia de reivindicación y articulación política de aquellos sujetos no reconocidos por las normativas. En un marco de fortalecimiento de los denominados nuevos movimientos sociales, desde finales de los años 80, las políticas de la identidad han sido tácticas prioritarias para aquellos colectivos abocados a dar batalla contra los sistemas de opresión como el sexismo, racismo, clasismo y la heterosexualidad obligatoria (Curiel, 2004). Particularmente, existen dos posturas divergentes en torno a la temática de las identidades, debates aún presentes dentro del campo feminista. A grandes rasgos, por un lado se encuentran los que consideran que las identidades son ficciones represivas y, por otro lado, quienes apuestan al reconocimiento identitario como estrategia política. La primera línea nos alarma que la noción misma de identidad puede conllevar a esencialismos y universalidades que tienden a reforzar los estereotipos que se pretenden romper y deconstruir. La segunda línea postula que la reivindicación de las identidades puede ser una buena estrategia política a partir de una reafirmación subjetiva que apunte a la transformación social (Curiel, 2004). Para concluir este trabajo, señalamos que no se ha pretendido polemizar si las identidades de género son, o no, una adecuada herramienta política, eso irá por cuenta de quienes lean. En tal caso, lejos de clausurar la temática, se intentó contribuir con reflexión teórica que invite a considerar al género como una construcción performativa (Butler, 1997 y 2001), pero sin perder de vista que también debería ser analizado de manera interseccional en relación con otros sistemas sociales de opresión como la clase social o la raza, de acuerdo con cada contexto en particular (Davis, 1981 y Viveros, 2006). En ello ha radicado la puesta en diálogo de estas dos grandes vertientes teóricas: el postestructuralismo y la interseccionalidad, ambas robustecedoras del inagotable camino de la lucha feminista. De este modo, las agendas del feminismo en los países latinoamericanos nos invitan a engendrar profundas discusiones sobre cuestiones teóricas, que atañen tanto al postestructuralismo como a las teorías poscoloniales, interpelando el lugar de la producción de conocimiento desde una perspectiva Sur-Sur (de Sousa Santos, 2009).

En ese recorrido, este trabajo ha intentado ayudar a trastocar algunas certezas ontológicas, lo que resulta fundamental a la hora de construir un pensamiento crítico que logre instalar a América Latina (y a los denominados países del sur) como lugares legítimos de enunciación discursiva y de conocimiento, pero sin aspiración universalista. 


\section{BIBLIOGRAFÍA}

Arfuch, Leonor (comp.) (2002): Identidades, sujetos y subjetividades. Buenos Aires: Prometeo.

Austin, John ([1962] 1992): How to do things with words. Cambridge: Harvard University Press.

Bidaseca, Karina (2010): Perturbando El texto colonial. Los estudios (pos) coloniales en América Latina. Buenos Aires: Editorial SB.

Butler, Judith (1997): "Sujetos de sexo / género / deseo". Feminaria. Año X, №19, Junio, Buenos Aires.

- (2001): El género en disputa. El feminismo y la subversión de la identidad. Buenos Aires: Paidós.

- (2004): Deshacer el género. Buenos Aires: Paidós.

--------- (2006) Vida precaria. El poder del duelo y la violencia. Buenos Aires: Paidós.

Braidotti, Rosi (1999): "Diferencia sexual y nomadismo". Revista Mora 5.

Crenshaw, Kimberlé (1994): "Cartographies des marges: intersectionnalité, politiques de I'dentité et violences contre les femmes de couleur"en Les Cahiers du Genre, Nro. 39.

Curiel, Ochy (2004): "Identidades esencialistas o construcción de identidades políticas: El dilema de las feministas negras" en: http://www.ciudaddemujeres.com/articulos/ IMG/pdf/Ochy_Curiel.pdf

Davis, Angela (1981): Mujeres, raza y clase Madrid: Akal.

De Beauvoir, Simone (1949): El segundo sexo. Buenos Aires: Sudamericana.

De Sousa Santos, Boaventura (2009): Una epistemología del Sur. México: CLACSO y Siglo XXI.

Derrida, Jacques (1971b): "La différance". In: WV.AA. Teoría de conjunto. Barcelona: Seix Barral.

-------- (1989): La Escritura y la Diferencia. Barcelona: Anthropos.

Fanon, Frantz (1963): Los condenados de la tierra. México: FCE.

Foucault, Michel (2003): Historia de la sexualidad. Vol. I: La voluntad del saber. Buenos Aires: Siglo XXI Editores. 
Fraser, Nancy (1997): Iustitia Interrupta. Reflexiones críticas desde la posición "postsocialista", Bogotá: Siglo del Hombre Editores/Universidad de los Andes, Santa Fe.

Gamba, Susana (coord.) (2007): Diccionario de estudios de género y feminismos. Buenos Aires: Biblos.

Hall, Stuart (1996):"Who needs identity?" en S. Hall \& P. Du Gay (eds.) Questions of cultural identity London: Sage.

Lacan, Jacques (1977): "El Seminario de Jacques Lacan"Libro 24, L'Insu, CD-Room obras completas, Folio View.

Rubin, Gayle (1993): El tráfico de mujeres. Notas sobre la economía política de los sexos. México: PUEG.

Tapalde Mohanty, Chandra (1988): "Bajo los ojos de Occidente. Academia Feminista y discurso colonial" en Boundary 212 no. 3/13, no. 1 (primavera / otoño).

Viveros, Mara (2002): De quebradores y cumplidores. Bogotá: CES-Universidad Nacional de Bogotá.

(2006): "El machismo latinoamericano. Un persistente malentendido"en Viveros et al. (Comp.) De mujeres, hombres y otras ficciones. Bogotá: Centro de Estudios Sociales - Escuela de Estudios de Género - Universidad Nacional de Colombia Tercer Mundo. Bogotá.

Zambrini, Laura e ladevito, Paula (2009): "Feminismo filosófico y pensamiento postestructuralista: Teorías y reflexiones acerca de las nociones de sujeto e identidad femenina" en Sexualidad, Salud y Sociedad - Revista Latinoamericana - CLAMUniversidad Estadual de Río de Janeiro. 